\title{
Verso la trasformazione del patrimonio edilizio italiano in NZEBs (Nearly Zero Energy Building): il progetto PRIN 2015
}

\author{
Towards the Italian building heritage transformation \\ into NZEBs (Nearly Zero Energy Building): \\ the PRIN 2015 project
}

\section{Laura Pompei}

Dipartimento di Ingegneria Astronautica, Elettrica ed Energetica

Università degli Studi di Roma La Sapienza

Via Eudossiana 18,00184 Roma

laura.pompei@uniromal.it

3334324155

\section{Sommario}

L'interesse dell'Unione Europea per la realizzazione di edifici energeticamente sostenibili è stato concretizzato attraverso la pubblicazione di molti documenti internazionali, tra cui la Direttiva europea 2010/31/UE, nota come EPBD Recast, che stabilisce che a partire dal 2021 tutti gli edifici di nuova costruzione debbano essere ad energia quasi zero (Nearly Zero Energy Building, NZEB), promuovendo anche la riqualificazione del patrimonio edilizio esistente in ottica NZEB. In questo quadro, il PRIN 2015 (Progetto di Ricerca di Interesse Nazionale) "Riqualificazione del parco edilizio esistente in ottica NZEB (nearly Zero Energy Buildings): costruzione di un Network Nazionale per la Ricerca" ha svolto un ruolo importante nell'elaborazione di molteplici soluzioni per la trasformazione degli edifici esistenti in NZEB, mettendo in luce i progressi raggiunti e i possibili sviluppi futuri.

\section{Parole chiave:}

$>$ NZEB

- Qualità ambientale interna

- Simulazione dinamica

- Risparmio energetico

\section{Abstract}

The European Union interest on the sustainable building realization has been actualized by several international normative, especially by the 2010/31/UE (Energy Performance of Buildings Directive) directive Recast. This directive establishes that starting from 2021 all new buildings must be a nearly Zero Energy Building (NZEB). Moreover, EPBD Recast underlines the importance of promoting the refurbishment of existing buildings to comply with the NZEB requirements. In this framework, the PRIN 2015 project (Progetto di Ricerca di Interesse Nazionale) called "Riqualificazione del parco edilizio esistente in ottica NZEB (Nearly Zero Energy Buildings): costruzione di un Network Nazionale per la Ricerca", played an important role in developing multiple solutions for the transformation of existing buildings into NZEBs, highlighting the research progress obtained and possible future developments.

\section{Keywords:}

$>$ NZEB

Indoor Environmental Quality

Dynamic simulation

$>$ Energy saving 


\section{Introduzione}

Negli ultimi decenni del secolo scorso, la necessità di costruire edifici energeticamente sostenibili si è affermata sia in Italia che in gran parte dei Paesi europei. L'Unione Europea ha infatti provveduto a concretizzare queste esigenze in termini sia progettuali che legislativi in modo tale da sensibilizzare tutti i Paesi a seguire specifiche Linee Guida. La direttiva principale, che ha segnato la svolta in questo campo, è la 2002/91/EC (Parlamento europeo, 2002), nota come Direttiva EPBD, Energy Performance of Buildings Directive, modificata poi con la Direttiva 2010/31/UE, nota come EPBD Recast (Parlamento europeo, 2010) e con la Direttiva (UE) 2018/844 (Parlamento europeo, 2018)). Secondo l'art. 2 della Recast, un Nearly Zero Energy Building, NZEB, è "un edificio ad altissima prestazione energetica, il cui fabbisogno risulta molto basso o quasi nullo ed è coperto in misura significativa da energia da fonti rinnovabili prodotta all'interno del sistema in loco o nelle vicinanze" (Parlamento europeo, 2010). Tutti gli Stati Membri hanno dovuto quindi provvedere affinché gli edifici di nuova costruzione occupati da enti pubblici fossero a energia quasi zero a partire dall'1 gennaio 2019 e tutti gli altri dall'1 gennaio 2021, così da promuovere anche la trasformazione del patrimonio edilizio esistente in ottica NZEB. In Italia il recepimento della EPBD è il D.Lgs. 192/2005, (Governo italiano, 2005) più volte modificato e integrato fino al 2015, con l'entrata in vigore del D.M. 26 giugno 2015 "Requisiti minimi" (MiSE, 2015). Seguendo le indicazioni della Direttiva (UE) 2018/844 (Parlamento europeo, 2018), le strategie nazionali per raggiungere le prestazioni di edifici ad alta efficienza e trasformare gli edifici esistenti in NZEB sono legate a tre aspetti principali: I'uso di strategie passive e l'applicazione di tecnologie ad alta efficienza energetica e di sistemi di generazione di energia rinnovabile (Harkouss et al., 2018). In questa ottica, il Progetto di Ricerca di Interesse Nazionale (PRIN) 2015 “Riqualificazione del parco edilizio esistente in ottica NZEB (nearly Zero Energy Buildings): costruzione di un Network Nazionale per la Ricerca", finanziato dal MIUR, ha avuto come obiettivo quello di studiare molteplici soluzioni per la trasformazione di edifici esistenti in NZEB, considerando le implicazioni sia tecniche che economiche. A questo progetto hanno partecipato sei unità di ricerca, appartenenti a diverse università italiane, ciascuna con competenze specifiche nei diversi aspetti di interesse per gli NZEB.

Lo scopo di questo lavoro è quello di mettere in luce gli avanzamenti raggiunti all'interno del progetto di ricerca e i possibili scenari di investigazione futura. L'articolo affronta tre macro tematiche di fondamentale importanza per gli NZEB: gli strumenti di simulazione, il risparmio energetico ed economico relativo al settore edilizio e la qualità dell'ambiente interno. Nello specifico, la prima tematica

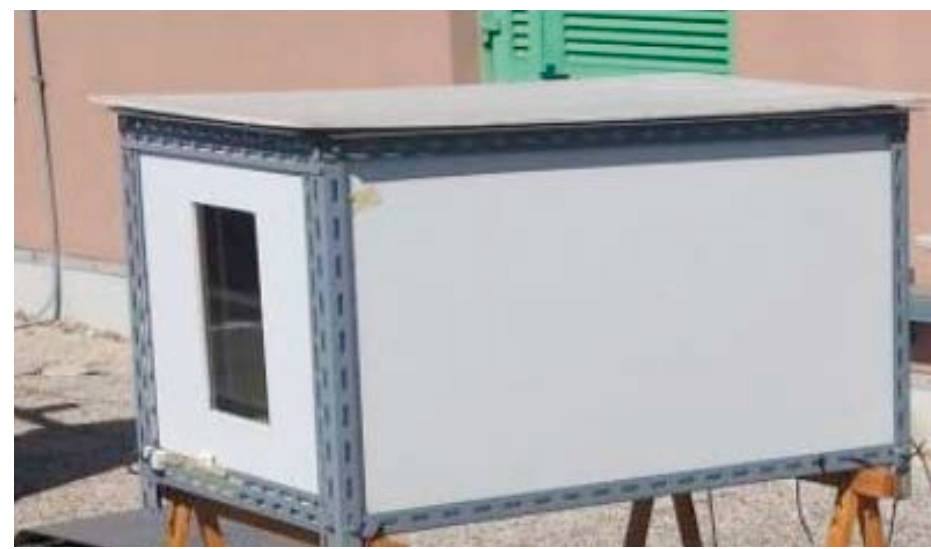

Figura 1 - Test Box Solare, STB, convenzionale in (Mazzeo et al., 2019)

Figure 1 - Solar Test Box, STB, conventional in (Mazzeo et al., 2019) approfondisce lo studio e la comparazione dei software di simulazione energetica, utilizzando dati reali provenienti da diversi casi studio; la seconda descrive diversi approcci di lavoro per la riduzione del fabbisogno energetico, dei costi di intervento e degli impatti delle diverse zone climatiche e la terza, infine, analizza i diversi metodi per la valutazione e il miglioramento della qualità dell'ambiente interno in termini acustici, termici e illuminotecnici.

\section{Metodologia}

\section{Modellazione dinamica e calibrazione del modello di edificio}

La simulazione dinamica degli edifici è diventata uno strumento consolidato per la previsione del comportamento termico dell'edificio. In quest'ottica, la ricerca è stata concentrata sulla valutazione dell'accuratezza dei software TRNSYS, EnergyPlus e IDA ICE, mediante il confronto tra i risultati simulati e le misurazioni sperimentali reali, evidenziandone le sostanziali differenze. In primo luogo, è stata effettuata una comparazione di tipo sperimentale tra un Test Box Solare, STB, convenzionale, in Figura 1, e uno basato sull'utilizzo dei Phase Change Material, i PCM (Mazzeo et al., 2019). Gli STB, situati presso I'Università di Roma Tor Vergata, sono provvisti di una stazione meteorologica che acquisisce i dati esterni reali necessari alle simulazioni. Partendo da questi dati rilevati, è stato possibile modellare gli STB con i tre software, seguendo la prassi di ciascun programma. Oltre alla comparazione con dati sperimentali, sono state effettuate alcune considerazioni sulla calibrazione di modelli dinamici di edifici esistenti cui hanno collaborato i gruppi di ricerca di PoliTO, PoliMI, UniTN, UniTOV (Angelotti et al., 2019), utilizzando come caso studio un edificio residenziale, riportato in Figura 2. Due gruppi di ricerca hanno utilizzato la calibrazione manuale, basata sull'analisi di sensibilità rispetto alle proprietà dell'involucro e ai parametri di comportamento degli occupanti, mentre gli altri due gruppi hanno utilizzato la calibrazione automatica, mediante algoritmi di ottimizzazione.

\section{Risparmio energetico ed economico nel settore dell'edilizia}

In generale, la previsione della domanda e del consumo di energia, sia su micro che su macro scala, sono innegabilmente strumenti fondamentali per la gestione dell'energia (Lorenzati et al., 2019; Mancini et al., 2019). Di conseguenza, risulta interessante investigare sia le modalità di accuratezza della fatturazione delle fonti di energia quali il Gas Naturale, GN, sia l'influenza di alcuni parametri nel calcolo del fabbisogno energetico avente grande impatto nelle prestazioni economico-finanziarie dell'edificio (Asdrubali et al., 2019). Pertanto, la ricerca ha condotto un'analisi comparativa tra i profili di carico standard per

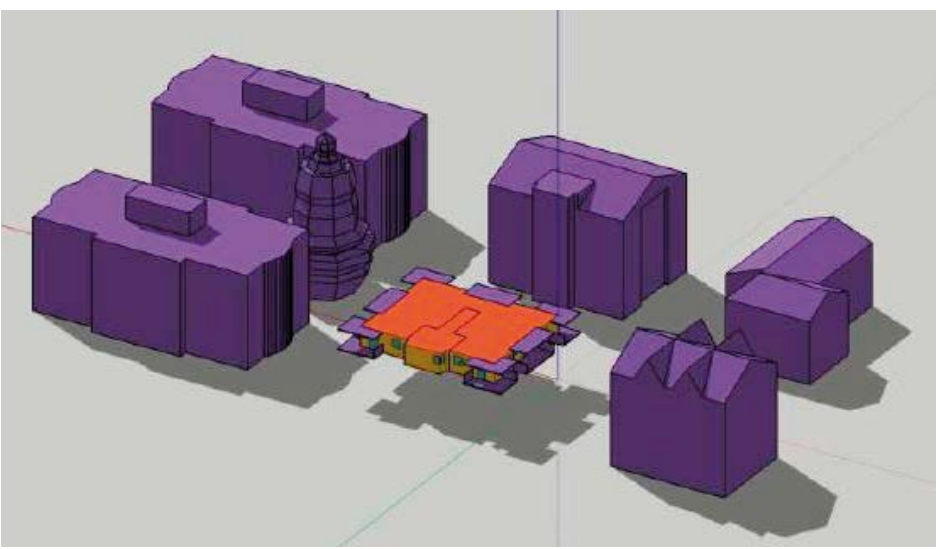

Figura 2 - Caso di studio dell'edificio residenziale in (Angelotti et al., 2019)

Figure 2 - Residential building case study in (Angelotti et al., 2019) 


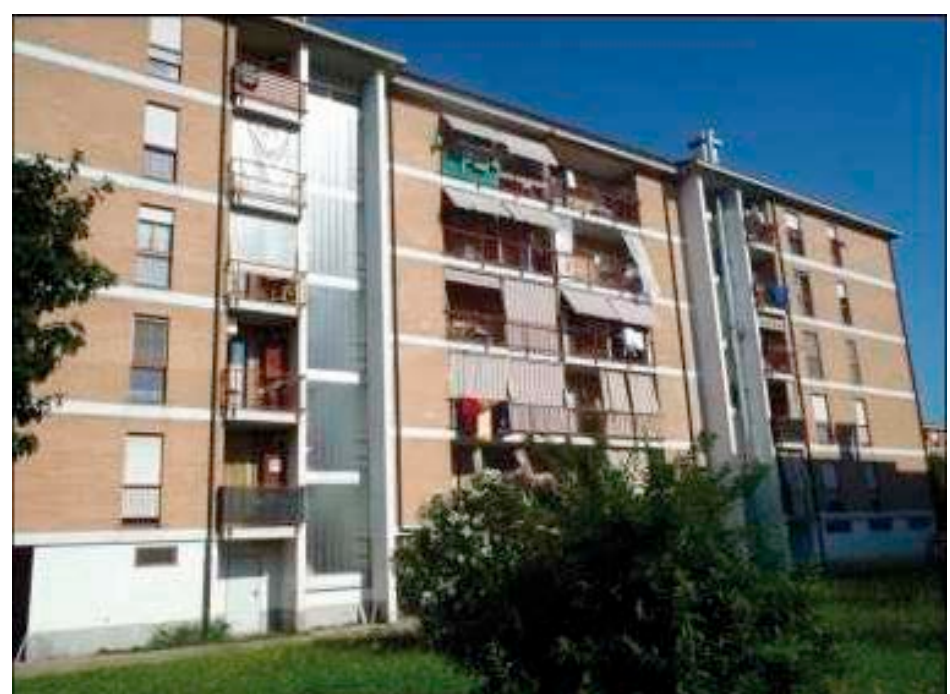

Figura 3 - Caso di studio edificio residenziale situato a Torino, Italia, in (Lorenzati et al., 2019)

Figure 3-Residential building case study located in Turin, Italy, in (Lorenzati et al., 2019)

la simulazione del consumo di GN su scala urbana (Canale et al., 2019): i ricercatori hanno studiato tre diversi metodi di allocazione del GN in Italia, Germania e Regno Unito con l'obiettivo di valutarne l'accuratezza dell'utilizzo per prevedere efficacemente il consumo di energia di un patrimonio edilizio relativo a due diverse scale spaziali, città e quartiere. Un altro fattore decisivo per la costruzione del fabbisogno energetico annuale è dato dalla valutazione della temperatura del cielo e della sua influenza in relazione alle diverse aree geografiche in cui l'edificio in osservazione è collocato (Asdrubali et al., 2019). Per questo tipo di analisi sono state considerate tre diverse condizioni climatiche (temperato mite: Roma, nevoso: Vienna e secco: Tripoli) e alcune correlazioni presenti in letteratura, che sono state implementate in TRNSYS; i risultati ottenuti sono stati confrontati con i valori di temperatura del cielo ottenuti applicando le correlazioni semplificate suggerite dalla norma UNI EN ISO 52016-1(UNI, 2018a).

In ambito NZEB, inoltre, le strategie di riqualificazione degli edifici esistenti assumono un ruolo rilevante, soprattutto nel settore edilizio residenziale (Lorenzati et al., 2019). Di conseguenza, sono state identificate due diverse configurazioni di intervento, la soluzione costoottimale (minimizzazione dei costi) e la soluzione NZEB (aumento dei livelli di prestazione termica e maggiore sfruttamento delle fonti di energia rinnovabile), applicate a un edificio residenziale situato nella provincia di Torino, in Figura 3, utilizzando il software DesignBuilder, interfaccia di EnergyPlus. Anche la previsione della domanda di energia ha ottenuto un forte impatto in questo ambito (Mancini et al., 2019): nello specifico è stata dimostrata l'opportunità di implementare un programma domanda/risposta attraverso la simulazione energetica di un campione di edifici. Di conseguenza, sono state definite le caratteristiche di tre kit di controllo (Low, Medium, High), in base all'incidenza dei servizi sui consumi energetici e un'analisi di sensibilità su alcuni driver energetici. Utilizzando la procedura stabilita dalla norma UNI EN 15232-1 (UNI, 2017a), sono stati valutati i risparmi energetici ed economici ottenibili. Infine, è stata effettuata un'analisi finanziaria degli investimenti.

\section{Qualità interna degli edifici basata sul comfort termico, acustico-visivo}

La qualità ambientale interna deve essere considerata parte integrante delle prestazioni energetiche dell'edificio, così come previsto dalla Direttiva 2018/844 (Parlamento europeo, 2018), garantendo la conformità ai requisiti di qualità dell'ambiente interno che sono definiti dal punto di vista oggettivo in termini acustici, dalle norme UNI

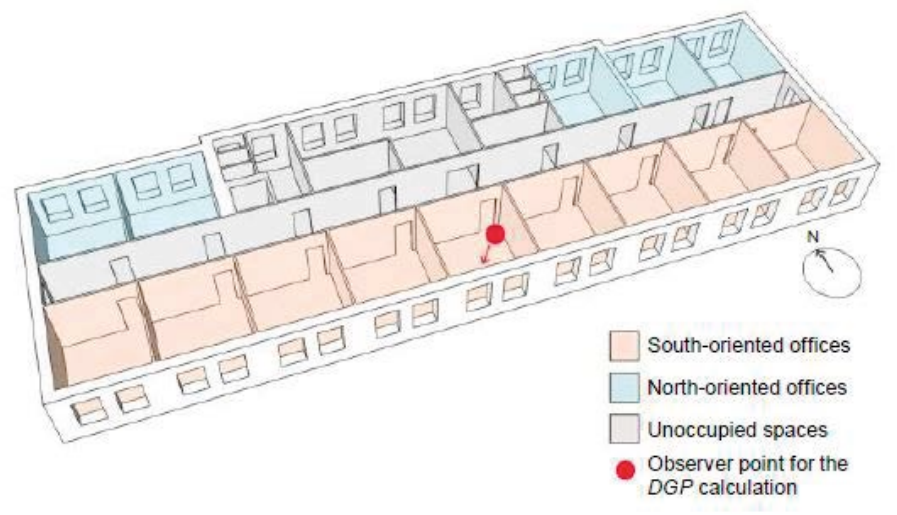

Figura 4 - Caso di studio in (De Luca et al., 2019)

Figure 4-Case study in (De Luca et al., 2019)

EN ISO 12354-1 (UNI, 2017b) e UNI EN ISO 16283-1 (UNI, 2018b), visivi, dalla norma UNI EN 12464 (UNI, 2011) e termici, dalla norme UNI EN ISO 7730 (UNI, 2006); questi stessi requisiti sono poi definiti dal punto di vista soggettivo dalla norma UNI EN ISO 10551 (UNI, 2019).

Per quanto riguarda le prestazioni termiche e visive, un primo filone della ricerca (De Luca et al., 2019) ha analizzato diverse combinazioni di simulazioni numeriche, utilizzando EnergyPlus e Diva4Rhino, entrambi software di simulazione dinamica. Gli indici di comfort termico e visivo sono stati calcolati secondo standard tecnici e riferimenti internazionali e come caso di studio è stato utilizzato un edificio esistente a uso ufficio, situato a Torino, in Figura 4, al quale sono state applicate tre strategie di gestione dell'ombreggiamento per il controllo della luce naturale durante la stagione calda, quella intermedia e quella fredda.

Un altro filone della ricerca (Bellia et al., 2019), che ha riguardato la relazione tra comfort termico e visivo, ha mostrato che i risultati delle indagini di laboratorio sono in buon accordo con la Hue-HeatHypothesis, $\mathrm{HHH}$, secondo cui la temperatura di colore correlata, CCT, può influire sulla percezione termica delle persone. I risultati delle simulazioni dinamiche di luce diurna e quelli delle sperimentazioni condotte presso il Laboratorio di Ingegneria Industriale dell'Università di Napoli Federico II, in Figura 5, hanno evidenziato che specifiche strategie di progettazione dell'ambiente interno luminoso possono modificare la percezione dell'ambiente termico e ridurre i consumi energetici dovuti all'illuminazione. La modellazione è stata eseguita con DIVA per Rhinoceros.

In un ulteriore filone della ricerca (Mastino et al., 2019), sono stati considerati i processi BIM per lo studio delle prestazioni acustiche degli

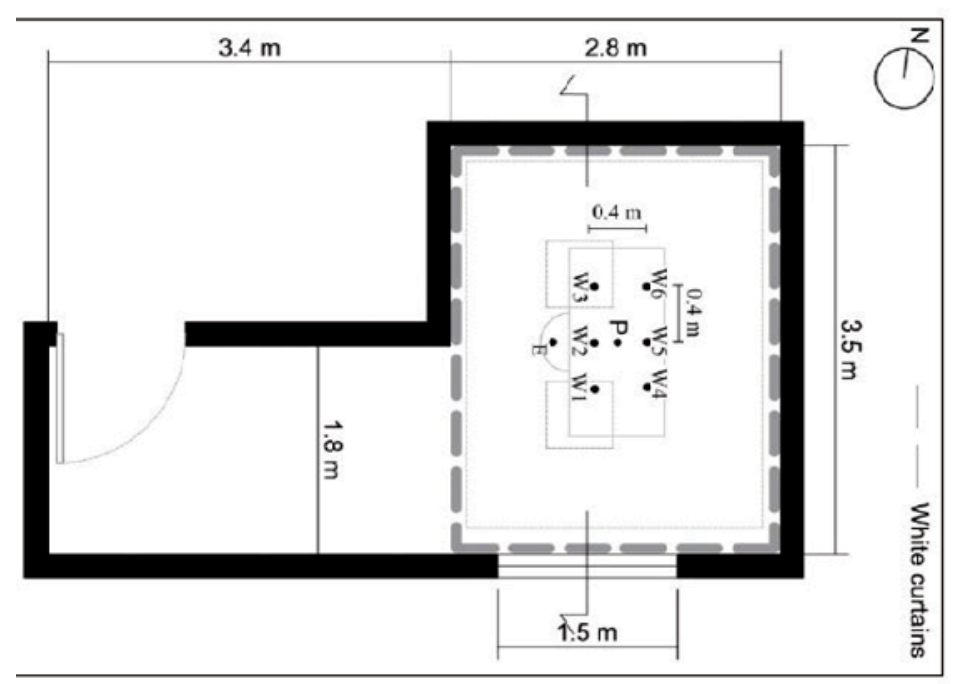

Figura 5 - Caso di studio in (Bellia et al., 2019)

Figure 5 - The test-room in (Bellia et al., 2019) 
edifici, implementando un nuovo codice di calcolo che consente la verifica dei limiti legislativi e l'integrazione dei dati post-costruzione registrati strumentalmente; tale codice è stato testato su un caso di studio monitorato nelle diverse fasi di costruzione.

\section{Risultati e discussione}

In questa sezione vengono presentati alcuni risultati significativi ottenuti, con riferimento ai punti descritti in ciascun paragrafo della metodologia.

\section{Modellazione dinamica e calibrazione del modello di edificio}

In (Mazzeo et al., 2019), sia in assenza che in presenza di PCM, gli strumenti Building Performance Simulation, BPS, mostrano un buon accordo con le tendenze sperimentali: le principali deviazioni tra i tre strumenti di BPS possono essere apprezzate soprattutto nel mese di dicembre a causa delle diverse ipotesi formulate nella definizione del modello. Confrontando i flussi termici emerge che EnergyPlus stima una maggiore dispersione di energia termica rispetto a TRNSYS e IDA ICE, portando a risultati meno accurati in assenza di PCM e che la precisione di EnergyPlus è quasi comparabile a quella di IDA ICE, il cui modello di isteresi entalpica consente di ottenere la migliore predizione del comportamento termico del STB provvisto di PCM. La Type 1270 di TRNSYS è, tra i modelli considerati, quella che porta alla peggiore accuratezza; tuttavia, sebbene non consenta di prevedere esattamente gli andamenti della temperatura durante il cambiamento di fase, consente di calcolare abbastanza accuratamente il calore latente complessivo immagazzinato. Relativamente alla calibrazione dei modelli dinamici (Angelotti et al., 2019), gli output dei diversi strumenti di simulazione sono generalmente coerenti tra loro: rispetto al profilo misurato, tutti sopravvalutano il valore medio della temperatura dell'aria interna anticipando i picchi. L'analisi di sensibilità eseguita da PoliTO e PoliMI ha mostrato una modesta influenza dei parametri esterni dell'involucro opaco. La calibrazione automatica eseguita da UniTOV, utilizzando IDA-ICE, ha prodotto un modello calibrato in linea con i dati misurati, che risulta più efficace della calibrazione manuale. Al contrario, la calibrazione manuale si traduce facilmente in diverse possibili soluzioni con prestazioni comparabili e con una riduzione del $24-25 \%$ del guadagno energetico giornaliero.

\section{Risparmio energetico ed economico nel settore dell'edilizia}

I modelli esaminati nel lavoro (Canale et al., 2019) e applicati su scala urbana presentano errori elevati, che tuttavia sono stati livellati su base annua, con conseguente loro riduzione. Inoltre, tutti i metodi hanno sottovalutato notevolmente il consumo di energia misurato del patrimonio edilizio considerato, a causa di un clima eccezionalmente freddo che si è verificato in Europa nel periodo considerato. Il metodo italiano ha prestazioni migliori rispetto agli altri, risultato prevedibile poiché tutti i modelli sono applicati a una rete energetica urbana italiana. I ricercatori di UNICAS hanno applicato il metodo italiano anche a livello di quartiere, simulando il consumo di energia di cinque edifici e il risultato è stato che riducendo la scala di applicazione del modello a un numero limitato di edifici, l'errore aumenta considerevolmente, a causa della mancanza di effetti di compensazione, ed è più elevato nella stagione estiva.

Per quanto riguarda lo studio sull'influenza della temperatura del cielo (Asdrubali et al., 2019), I'implementazione in TRNSYS dell'equazione semplificata fornita da UNI EN ISO 52016-1 (UNI, 2018a). o di uno specifico modello di temperatura di cielo fittizia può portare a differenze di temperatura significative, in condizioni di cielo sia sereno che nuvoloso. Inoltre, tenendo conto degli effetti derivanti dall'impiego di diverse correlazioni nelle simulazioni energetiche degli edifici, è stato riscontrato che i fabbisogni energetici di riscaldamento e raffrescamento possono essere influenzati notevolmente dalle condizioni climatiche miti registrate a Roma, nevose proprie del clima di Vienna, e secche di Tripoli. Le Misure di Efficienza Energetica riportate in (Lorenzati et al., 2019) sono state selezionate per identificare la soluzione di recupero energetico caratterizzata dal più basso costo globale per un periodo di 30 anni. È stato osservato che la soluzione costo-ottimale non soddisfa i requisiti NZEB, per cui è stata selezionata una nuova configurazione, caratterizzata da una combinazione di strategie di efficientamento riguardanti l'involucro, quali schermature e isolamento termico esterno, e i sistemi impiantistici, ad esempio caldaie a condensazione e pompe di calore, includendo l'utilizzo di impianti fotovoltaici. La soluzione NZEB così identificata consente di dimezzare il consumo di energia in termini di energia primaria non rinnovabile rispetto alla soluzione costo-ottimale, causando quasi un raddoppio del costo totale dell'investimento rispetto a quello dell'attuale edificio.

L'applicazione del programma domanda/risposta di (Mancini et al., 2019) per gli utenti residenziali può consentire di aumentare la flessibilità della domanda, facilitando la diffusione di energie rinnovabili; tuttavia, mostra significative complessità di implementazione dovute alla frammentazione dei carichi. Utilizzando il kit di controllo Low, i risparmi di energia primaria sono in media del 5,3\%, mentre i risparmi economici sono in media pari al 9,2\%; utilizzando il kit di automazione Medium, i risparmi di energia primaria sono del 10,8\% e i risparmi economici del 14,6\%; utilizzando il kit High, i risparmi di energia primaria sono dell'11,7\% e i risparmi economici del 15,5\%. L'analisi finanziaria sugli investimenti indica che il tempo di ritorno è inferiore a 10 anni.

\section{Qualità interna degli edifici basata sul comfort acustico, termico e visivo}

Tra le tre combinazioni analizzate in (De Luca et al., 2019) per il caso di studio, quella ottimale di comfort termico mostra le peggiori condizioni di comfort visivo sia per quanto riguarda la disponibilità di luce diurna sia per il disagio dovuto all'abbagliamento; allo stesso modo, la combinazione ottimale di comfort visivo porta a valori molto alti del WHDw, Warm Discomfort index, e di richiesta di energia primaria complessivi, a causa di un aumento dei consumi energetici per il riscaldamento e il raffrescamento. La scelta della soluzione ottimale per il caso di studio considerato conferma la necessità di individuare una strategia di controllo che combini i requisiti di comfort termico e comfort visivo, contribuendo anche a migliorare le prestazioni energetiche dell'edificio.

Sulla base dei risultati ottenuti da (Bellia et al., 2019), in condizioni controllate di illuminazione e di parametri termoigrometrici, è possibile confermare che, in condizioni invernali la luce più fredda (6000 K) induce uno spostamento della sensazione termica verso il freddo. L'effetto sembra essere più pronunciato nel caso di donne quando si varia la CCT da $3000 \mathrm{~K}$ (luce calda) a $6000 \mathrm{~K}$ (luce fredda). Le simulazioni dinamiche di luce diurna dimostrano che le strategie volte ad applicare l'HHH influenzano non solo le caratteristiche dell'ambiente luminoso, ma anche i consumi energetici dovuti all'illuminazione.

Gli aspetti più importanti emersi nei test sperimentali di (Mastino et al., 2019) consistono nell'evidenziare il ruolo fondamentale che il sistema di codifica deve garantire per l'interoperabilità tra i diversi strumenti di lavoro del software utilizzato. Il controllo del codice eseguito sul caso di studio ha mostrato come le indagini strumentali riescano a mettere in evidenza le caratteristiche acustiche dell'edificio durante tutte le fasi di costruzione e collaudo, migliorando il risultato finale in termini di qualità. 


\section{CONCLUSIONI}

Il progetto PRIN 2015, finanziato dal Ministero dell'Istruzione, dell'Università e della Ricerca (MIUR), ha interessato un arco temporale della durata di tre anni, nei quali tutte le unità operative coinvolte hanno contribuito alla realizzazione di nuove soluzioni per la tematica NZEBs. Durante l'ultimo anno è stato possibile raggruppare le diverse ricerche nelle seguenti tematiche: la modellazione dinamica e la calibrazione del modello di edificio, il risparmio energetico ed economico nel settore dell'edilizia, la qualità ambientale interna degli edifici basata sul comfort acustico, termico e visivo. II progetto ha anche investigato molti aspetti dei più noti e utilizzati software dinamici, analizzando anche nuovi strumenti quali il BIM.

È stato quindi possibile modellare diversi casi di studio, relativi all'edilizia residenziale, per investigare possibili scenari di trasformazione NZEB, sia energetici che economici. In ambito nazionale il progetto qui sintetizzato, insieme a un altro progetto PRIN intitolato "Supporto alle Municipalità per i PAESC (Piani di Azione per l'Energia Sostenibile e il Clima)", ha ottenuto una menzione speciale dal Ministero per la Semplificazione a la Pubblica Amministrazione, d'intesa con il Ministero dell'Istruzione, dell'Università e della Ricerca, nell'ambito del concorso "ITALIA DECIDE". A livello internazionale, gli ultimi progressi del progetto sono stati presentati alla conferenza "Building Simulation 2019", tenutasi a Roma nel mese di settembre, nel corso della quale le università partecipanti (UNIROMA, UNICAS, IUAV, UNISA, POLITO, POLIMI, UNITOV, UNICA, UNITN, UNINA, UNICAL e UNIROMA3) hanno condiviso con la comunità scientifica proveniente da tutto il mondo i risultati ottenuti dal progetto di ricerca e presentato gli sviluppi futuri per la realizzazione di strutture a energia zero (ZEB, zero energy building).

CONFLITTO DI INTERESSE

Gli autori dichiarano che non esistono conflitti di interesse di ordine economico o di altro tipo sull'articolo presentato.

\section{FINANZIAMENTI}

Questo lavoro rientra nel quadro del progetto "Riqualificazione del parco edilizio esistente in ottica NZEB (nearly Zero Energy Buildings): costruzione di un Network Nazionale per la Ricerca" che ha beneficiato del sostegno finanziario del MIUR attraverso il programma PRIN 2015.

\section{BIBLIOGRAFIA}

Angelotti A., Ballabio M., Mazzarella L., Cornaro C., Parente G., Frasca F., Prada A., Ballarini.l, Del Luca G., Corrado V. 2019. Dynamic simulation of existing buildings: considerations on the model calibration. Proceedings of 16th IBPSA International Conference \& Exhibition Building Simulation, Rome, September.

Asdrubali F., Evangelisti L., Grazieschi G., Guattari C. 2019. Influence of sky temperatures on building energy needs. Proceedings of 16th IBPSA International Conference \& Exhibition Building Simulation, Rome, September.

Bellia L., d'Ambrosio Alfano F.R., Fragliasso F., Palella B.I., Riccio G. 2019. Dynamic simulation of a lighting system based on the Hue-Heat Hypothesis. 16th IBPSA International Conference \& Exhibition Building Simulation, Rome, September.

Canale L., Cortellessa G., Dell'Isola M., Ficco G., Frattolillo A., Zuena F., Castaldi A. 2019. A comparative analysis among standard load profiles for natural gas consumption simulation at urban scale. Proceedings of 16th IBPSA International Conference \& Exhibition Building Simulation, Rome, September.

De Luca G., Ballarini I., Paragamyan A., Pellegrino A., Corrado V. 2019. Optimized solutions for thermal and visual comfort in the design of a nearly zero-energy building. Proceedings of 16th IBPSA International Conference \& Exhibition Building Simulation 2019.

Harkouss F., Fardoun F., Biwole P.H. 2018. Optimization approaches and climates investigations in NZEB - A review. Building Simualiton, 11(5), 923-952.

Lorenzati A., Ballarini I., De Luca G., Corrado V. 2019. Social housing in Italy: energy audit and dynamic simulation towards a NZEB policy. Proceedings of 16th IBPSA International Conference \& Exhibition Building Simulation, Rome, September.

Mancini F., Basso G.L., Santoli L.D. 2019. Energy use in residential buildings: Impact of building automation control systems on energy performance and flexibility. Energies, 12(15).

Mastino C., Baccoli R., Frattolillo A., Marini M., Salaris C. 2019. Acoustic insulation and building information modeling: a model of calculation for the 2 Code checking in the forecast phase and of measurement of performance. Proceedings of 16th IBPSA International Conference \& Exhibition Building Simulation, Rome, September.

Mazzeo D., Romagnoni P., Matera N., Oliveti G., Cornaro C., De Santoli L. 2019. Accuracy of the most popular building performance simulation tools: experimental comparison for a conventional and a PCM-based Test Box. Proceedings of 16th IBPSA International Conference \& Exhibition Building Simulation, Rome, September.

MiSE. 2015. Applicazione delle metodologie di calcolo delle prestazioni energetichee definizione delle prescrizioni e dei requisiti minimi degli edifici. D.M.26/06/2015 Gazzetta Ufficiale n. 162 del 15.07.2015, S.O. n. 39. Roma: Poligrafico dello Stato.) Parlamento europeo. 2010. Direttiva 2010/31/UE del Parlamento europeo e del Consiglio del 16 dicembre 2002 sul rendimento energetico nell'edilizia. Gazzetta ufficiale dell'Unione europea, L1/65 del 4.1.2003.

Parlamento europeo. 2010. Direttiva 2010/31/UE del Parlamento europeo e del Consiglio del 19 maggio 2010 sulla prestazione energetica nell'edilizia (rifusione). Gazzetta ufficiale dell'Unione europea, L153/13 del 18.06.2010.

Parlamento europeo. 2018. Direttiva (UE) 2018/844 (EPBD) del Parlamento europeo e del Consiglio del 30 maggio 2018 che modifica la direttiva 2010/31/UE sulla prestazione energetica nell'edilizia e la direttiva 2012/27/UE sull'efficienza energetica. Gazzetta Ufficiale dell'Unione Europea, L156/75 del 19.06.2018.

Governo italiano. 2002. Attuazione della direttiva 2002/91/CE relativa al rendimento energetico nell'edilizia. D.Lgs. 19 agosto 2005, n. 192. G.U. n. 222 del 23.09.2005.

UNI. 2006. Ergonomia degli ambienti termici - Determinazione analitica e interpretazione del benessere termico mediante il calcolo degli indici PMV e PPD e dei criteri di benessere termico locale. Norma UNI EN ISO 7730. Milano: Ente Italiano di Normazione.

UNI. 2011. Luce e illuminazione - Illuminazione dei posti di lavoro - Parte 1: Posti di lavoro in interni. Norma UNI EN 12464. Milano: Ente Italiano di Normazione.

UNI. 2017a. Prestazione energetica degli edifici - Parte 1: Impatto dell'automazione, del controllo e della gestione tecnica degli edifici - Moduli M10-4,5,6,7,8,9,1. Norma UNI EN 15232-1. Milano: Ente Italiano di Normazione.

UNI. 2017b. Acustica in edilizia - Valutazioni delle prestazioni acustiche di edifici a partire dalle prestazioni dei prodotti - Parte 1: Isolamento dal rumore per via aerea tra ambienti. Norma UNI EN ISO 12354-1. Milano: Ente Italiano di Normazione.

UNI. 2018a. Prestazione energetica degli edifici - Fabbisogni energetici per riscaldamento e raffrescamento, temperature interne e carichi termici sensibili e latenti - Parte 1: Procedure di calcolo. Norma UNI EN ISO 52016-1. Milano: Ente Italiano di Normazione.

UNI. 2018b. Acustica - Misure in opera dell'isolamento acustico in edifici e di elementi di edificio - Parte 1: Isolamento acustico per via aerea. Norma UNI EN ISO 16283-1. Milano: Ente Italiano di Normazione.

UNI. 2019. Ergonomia dell'ambiente fisico - Scale di giudizio soggettivo per la valutazione degli ambienti fisici. Norma UNI EN ISO 10551. Milano: Ente Italiano di Normazione. 